\title{
Minority Health and Health Disparities in the 21st Century: A Review
}

\author{
Devontenno K., Gary J., Grandville N., Hale D., Hayes A., Mosley M. and Mandal P* \\ Edward Waters University, 1740 Kings Road, Jacksonville, Florida.
}

*Corresponding Author: Mandal P, Professor of Biology, Edward Waters University, 1740 Kings Road, Jackson ville, 32209, Florida.

Received date: Augusł 03, 2021; Accepted date: September 07, 2021; Published date: September 18, 2021

Citation: Mandal P, Devontenno K., Gary J., Grandville N., and Hale D., et.al. (2021). Minority Health and Health Disparities in the 21st Century: A Review. J Clinical Research and Reports, 9(1); DOI:10.31579/2690-1919/194

Copyright: (c) 2021, Mandal P. This is an open access article distributed under the Creative Commons Attribution License, which permits unrestricted use, distribution, and reproduction in any medium, provided the original work is properly cited.

\begin{abstract}
Powerful, complex relationships exist between health and biology, genetics, and individualbehavior, and between health and health services, socioeconomic status, the physical environment, discrimination, racism, literacy levels, and legislative policies. These factors, which influence an individual's or population's health, are known as determinants of health. Today, health disparity is taking an in depth look at the differences in health status between different social groups, gender,race, ethnicity, education, income, disability, and sexual orientation. While on the other hand, health inequality is looking at the unjust and unfair treatment one gets because of their socioeconomic status and demographic area. Such a wide array of differences in health inequalityand disparity is what contributes to the United States ranking in the bottom of industrialized western nations when it comes to life expectancy rate, and infant mortality rate. Even though overthe years there have been great improvements and changes, there is still more work to be done to make health and equality for all.
\end{abstract}

Keywords: African Americans; disparity; education; health; minority; socioeconomic factors; chronic illness; medications; chronic lower respiratory disease

\section{Introduction}

The United States Healthcare or Medical care system is structured in such a way that its population is faces with many healthcare challenges, such as the high rising cost of healthcare, theneed for a strong public health work, persistent disparities among racial/ethnic minority groups aswell as underserved populations. Even with major medical advances, there are critical threats to the health of the United States population and with different health disparities trending (1). Apartfrom healthcare there are social factors that contribute tremendously to shape the health of populations within different environments. Braveman and Gottlieb , 2014 (2) mentioned a large and compelling body of evidence which has been accumulated over the last two decades revealed that healthcare is not the only influence on health, but it plays a major role due to it being limited.Evidence for this can be found in reports from the National Research Council and Institute of Medicine that the U.S health is detrimental in both morbidity and mortality across most health indicators and all age groups except for those over the age of 75; it is shown in both wealthy as well as poor Americans, also to non-Hispanic white people (2).

Some of the health conditions that minority groups with health disparities suffer among theleading cause of mortality both in the U.S and worldwide; they include cardiovascular disease, diabetes, asthma, and cancer, among other conditions. These disparities are cause by a group of factors, including environmental and or genetical factors, with the largest contributors being the social determinants of health external to the health care system. This can be seen within minority communities where members tend to be more socio-economically disadvantaged, to have lower levels of education, to work in jobs with higher rates of occupational hazards and to live in areas with greater environmental hazards, such as air pollutant, than members of the majority population. Among the many different root causes of the disparities that have been explored and presented, there are variations in patients' health beliefs, values, preferences, and behaviors which have recently gained much attention. They include the variations in patient recognition of symptoms; thresholds for seeking care; the ability to communicate symptoms to a provider who understands their meaning; the ability to understand the prescribed management strategy; expectations of care which includes the preferences for or against diagnostic and therapeutic procedures; and obedienceto precautionary measures and medications given. These factors are said to influence patient andphysician decisionmaking and the interactions between patients and the health care delivery system, which contributes to the health disparities (3).

Compared to the European Americans counterparts, African Americans are generally at higher risk for heart disease, stroke, cancer, obesity, asthma, influenza, pneumonia, diabetes, and HIV/AIDS. Also, 
when compared with men of other races and ethnicities, African American population have higher rates of mortality and morbidity from chronic illness and diseases. To improve the health and eliminate these conditions of Black and minority ethnic group, it is important to include them in health research, to elicit their perspectives on health risks and protective factors. These can then inform interventions aimed at reducing health disparities. However, there are still challenges remain in recruiting and engaging African American men in health research (4). Although not often considered as a health condition, obesity sits at the stop ofall because it is often the gate to many other health conditions such as hypertension, diabetes, cardiovascular disease as well as strokes. Even though obesity prevalence is stabilizing in the United States, nonHispanic African American and Hispanics remain disproportionately affected (5). The high impact on obesity contributes high risk of cardiovascular disease among the Hispanic and Black ethnic group; although hypertension is also highly prevalent among the Black community it contributes directly to the high burden of cardiovascular disease in the African American community (6).

\section{Discussion}

The question to ask is how do widespread and persistent socioeconomic gradients in healthadd to evidence that social factors are important influences on health? There have been some strong links observed for centuries between poverty and health. The observation of a graded relationship(as opposed to a simple threshold, for instance at the poverty level) of socioeconomic factors withmany different health indicators recommend a possible dose-response relationship, adding to the likelihood that socioeconomic factors, or factors that may be closely associated with them, play acausal role. Even though the effects of miserable poverty on health are rarely discussed, not everyone agree to the effects of income and level of education on health across the socioeconomic spectrum; Some may argue that income-health or education-health relationships reflect reverse causation, that is sickness leading to income loss and/or lower educational achievement. In Breveman and Gottlieb, 2014 (2) finding, it states that while ill health often results in the loss of income, and a child's poor health could limit educational achievement, evidence from longitudinaland cross-sectional studies indicate that these do not account for the strong, persistent relationshipsobserved. Though the links between education and health cannot be explained using reverse causation, because once attained, educational attainment is never reduced, there are evidence to show that majority of the minority population education is limited due to poor living conditions (2).

In a study that aimed to identify the self-reported health care experiences and outcomes fora general population of cultural minority Americans in the Midwestern state, the influences of cultural strength on health care encounters were examined. The participant in this study reported that staff knowledge on different cultures and diversity is important in the health care system. TheNative Americans reposted poor treatment and believed that cultural matching such be done in thehealth care services (7). The study conducted by Cuevas et al., 2016 (8) these discriminations in the health care setting among African American resulted when patients, mainly women, felt theirhealth issues were discredited; Also medical mistrust took placed when clinicians did not show respect to patients, leaving them to wonder whether their treatment were discriminatory or not; while poor communication arose when clinician did not acknowledge patients' perspectives duringinteractions, thus these are viewed as actions of discriminatory.

Sociodemographic environment, including neighborhood socioeconomic status and racial/ethnic composition, has been more consistently associated with obesity. Residents of low socioeconomical status communities are exposure to poor nutrient food, which leads to poor eatinghabit and bad health such as obesity. In a study that examine the associations between soda consumption and weight status, shown a positive correlation between the two. The study findingssuggest that associations between neighborhood environment characteristics and obesity-related behaviors and outcomes vary by race/ethnicity. Efforts to intervene on neighborhood-built environments might not benefit all racial/ethnic subgroups equally. Future research should explorepotential pathways to better understand the relationship between obesity outcomes and theneighborhood environment in different race/ethnicity groups, particularly in high-risk minority groups (5). The higher prevalence of traditional cardiovascular risk factors such as hypertension, diabetes mellitus, obesity, and atherosclerotic cardiovascular risk, underlies the relatively earlier age of onset of cardiovascular diseases among African Americans. Hypertension is highly prevalent among African Americans and contributes directly to the notable disparities in stroke, heart failure, and peripheral artery disease among African Americans (6).

\section{Conclusion}

Populations with a lower socioeconomic status, rural communities, people with cognitive and physical disabilities and are often disproportionately exposed to conditions and environmentsthat negatively affect health risks and outcomes and lead to higher rates of health disparities. For example, Americans living in rural areas are more likely to die from unintentional injuries, heart disease, cancer, stroke, and chronic lower respiratory disease than their urban counterparts. To address these disparities in health care access and quality is to bring more diversity to the ranks of health services researchers because their findings inform the policies and regulations that ultimately govern the delivery of health care. The need to increase the ethnic and racial diversity of the research workforce is widely recognized. Addressing the true underrepresentation of minorities in health services research will require pre-college programs as a long-term strategy tocomplement post-college efforts.

\section{References}

1. Jackson, C. S., and Gracia, J. N. (2014). Addressing health and health-care disparities: the role of a diverse workforce and the social determinants of health. Public health reports (Washington, D.C.: 1974), 129 Suppl 2 (Suppl 2), 57-61.

2. Braveman, P., and Gottlieb, L. (2014). The social determinants of health: it's time to consider the causes of the causes. Public health reports (Washington, D.C.: 1974), 129Suppl 2 (Suppl 2), 19-31.

3. Betancourt, J. R., Green, A. R., Carrillo, J. E., and AnanehFirempong, O. (2003).

4. Defining cultural competence: a practical framework for addressing racial/ethnic disparities in health and health care. Public health reports (Washington, D.C.: 1974),118(4), 293-302.

5. Randolph, S., Coakley, T., and Shears, J. (2018). Recruiting and engaging African American men in health research. Nurse researcher, $26(1), 8-12$.

6. Wong, M. S., Chan, K. S., Jones-Smith, J. C., Colantuoni, E., Thorpe, R. J., Jr, andBleich, S. N. (2018). The neighborhood environment and obesity: Understanding variation by race/ethnicity. Preventive medicine, 111, 371-377.

7. Carnethon, M. R., Pu, J., Howard, G., Albert, M. A., Anderson, C., Bertoni, A. G.,Mujahid, M. S., Palaniappan, L., Taylor, H. A., Jr, Willis, M., Yancy, C. W., and American Heart Association Council on Epidemiology and Prevention; Council on Cardiovascular Disease in the Young; Council on Cardiovascular and Stroke Nursing; Council on Clinical Cardiology; Council on Functional Genomics and Translational Biology; and Stroke Council (2017). Cardiovascular Health in African Americans: A Scientific Statement from the American Heart Association. Circulation, 136(21), e393-e423. 
8. Shepherd, S. M., Willis-Esqueda, C., Paradies, Y., Sivasubramaniam, D., Sherwood, J., and Brockie, T. (2018). Racial and cultural minority experiences and perceptions of health care provision in a mid-western region. International journal for equity in health, 17 (1), 33.
9. Cuevas, A. G., O'Brien, K., and Saha, S. (2016). African American experiences in healthcare: "I always feel like I'm getting skipped over". Health psychology: officialjournal of the Division of Health Psychology, American Psychological Association, 35(9), 987-995.
Ready to submit your research? Choose Auctores and benefit from:

$>$ fast, convenient online submission

$>$ rigorous peer review by experienced research in your field

$>$ rapid publication on acceptance

$>$ authors retain copyrights

$>$ unique DOI for all articles

$>$ immediate, unrestricted online access

At Auctores, research is always in progress.

Learn more auctoresonline.org/journals/journal-of-clinical-research-andreports 